\title{
SIMULTANEOUS MAGNETIC FIELD MEASUREMENTS IN SUNSPOTS USING SPECTRAL LINES WITH DIFFERENT LANDE FACTORS
}

\author{
S.N.Osipov ${ }^{1}$, V.G.Lozitsky ${ }^{2}$ \\ ${ }^{1}$ Main Astronomical Observatory of National Academy of Sciences, \\ Zabolotnoho 27, Kiev 03680, Ukraine,osipov@mao.kiev.ua \\ ${ }^{2}$ Astronomical Observatory of Taras Shevchenko National University of Kyiv, \\ Observatorna 3, Kyiv 01053, Ukraine, lozitsky@observ.univ.kiev.ua
}

\begin{abstract}
We present magnetic field measurements in several sunspots observed in June-July 2015 on Horizontal Solar Telescope ATsU-5 of Main Astronomical Observatory of National Academy of Sciences of Ukraine. The Zeeman splittings were measured using $I \pm V$ profiles of about ten spectral lines of $\mathrm{Mn} \mathrm{I}, \mathrm{Fe} \mathrm{I}$ and Ni I including three lines with negative Lande factors, namely Fe I $5434.527 \AA$, Fe I $6094.419 \AA$ and Fe I 4995.411 $\AA$ ( $g_{\text {eff }}=-0.014,-0.218$, and -0.25 , respectively). Our main conclusions are the following:

a) as rule, spectral lines with largest Lande factors give the strongest measured magntic fields $B_{\text {obs }}$ in sunspot umbra that can be interpreted as a result of blending the Zeeman $\pi$ - and $\sigma$-components in case of non-longitudinal magnetic field.

b) in some places of sunspots, $B_{\text {obs }}$ differs also for lines with close Lande factors, e.g. by Fe I 5432.950 and Ni I 5435.871 ( $g_{\text {eff }}=0.67$ and 0.5 , respectively).

c) lines Fe I $6094.419 \AA$ and Fe I $4995.411 \AA$ with $g_{\text {eff }}<0$ have in sunspots signs of splitting which corresponds to $g_{\text {eff }}>0$.
\end{abstract}

The possible causes of named effects are discussed in short form.

Keywords: Sun: Solar magnetic fields - Sunspots: direct measurements - Lande factors.

\section{Introduction}

Magnetic field measurements in different spectral lines allow obtain very important information about vertical and horizontal magnetic fields inhomogeneity in solar atmosphere. In case of homogeneous magnetic field, we can expect the same measured magnetic field strengths by different spectral lines. If magnetic field is inhomogeneous, measured field values can depend from depth of line formation, its temperature sensitivity, etc. Let us remember some important results to last case.

Gopasyuk et al. (1973) found very interesting effect for non-sunspots regions: measured magnetic field strength depends from magnetic sensitivity factor $g \lambda^{2}$. This effect, obtained by magnetographic method from analyze of Stokes $V$ parameter, was confirmed lately in Stokes $I$ using photographical method (Lozitsky, 1980). As it was argued in last paper, probable cause of this dependence is the presence of spatially unresolved magnetic fields with strength of 8-10 kG.

In the same year, Stenflo (1973) discovered the most important observational effect to existence of the spatially unresolved magnetic fields with $\mathrm{kG}$ fields in the quiet atmosphere. He used two spectral lines of the same multiplet (No. 1) of Fe I, 5247.1 $\AA$ and 5250.2 $\AA$, which have practically equal heights of formation and temperature sensitivity, but different Lande factors $\mathrm{g}_{\text {eff, }}$, 2.0 and 3.0, respectively. It was found that, in general, $B_{\text {obs }}(5247.1) \neq B_{\text {obs }}(5250.2)$. According to simulations, Stenflo's (1973) observations correspond to $B_{\text {true }}=1.1-2.3$ $\mathrm{kG}$ in spatially unresolved magnetic fluxtubes.

Semel (1981) had compared the measured magnetic fields $B_{\text {obs }}$ in 12 spectral lines using lambda-meter for observations of plages and sunspots. He found a good agreement of such fields for sunspots, but very essential differences (till 5 times) for plages.

Solanki and Stenflo (1984) studied the statistical properties of the Stokes $I$ and $V$ line profiles of 400 unblended Fe I lines in areas of photospheric network and plages. Observations were carried out at Kitt Peak McMath telescope using FTS technique. They concluded that true magnetic field $B_{\text {true }}$ in fluxtubes is typically 1.4 $1.7 \mathrm{kG}$, and the filling factor is $3-4 \%$ for network and 5$15 \%$ for plages. In addition, it was found that 12 lines of high multiplets (№№ 823-1177) have very different observed and theoretical (for LS coupling) Lande factors. As to Fe I $5434.527 \AA$ line $\left(g_{\text {eff }}=-0.014\right)$, authors had concluded: "Our data show no sign of this line in the $V$ spectra, consistent with a $g_{\text {eff }}$ of zero".

However, another effect was found later in this line. In spectra of powerful solar flares this line has sometimes appreciable splitting of narrow emissive peaks at its core that correspond to magnetic field strength of about several tens kG (see, e.g., Lozitsky, 2011, 2015).

The purpose of this paper is to analyze the new observational data on the Zeeman splitting in sunspots of different spectral lines, which have both well-known and "doubtful" Lande factors.

\section{Observations}

Observations were carried out on Horizontal Solar Telescope ATsU-5 of Main Astronomical Observatory of 
National Academy of Sciences of Ukraine. Spectra recordings were performed using the SBIG ST-8300 CCD camera. For observations of $I+V$ and $I-V$ spectra, the polarization mosaic made by V.I. Skomorovsky and quarter-wave plate were used. Measured spectra were corrected for flatfield, parasitic interference and curvature of spectral lines.

During June-July 2015, we observed several sunspots placed no far from disk center, in particular, the greatest spots on following dates: June 10, 20, 23 and 26, 2015 and July 08 and 18, 2015.

Stokes $I \pm V$ spectra were recorded in about $\pm 4 \AA$ ranges around following wavelengths: 4995.4, 5434.5 and 6094.4 ^ (Fig. 1). About 6-8 well-visible spectral lines with different Lande factors were observed in named wavelength ranges. In this short paper, we present the most obvious effects only, without many details of our study, which is planned to presentation in a separate article.

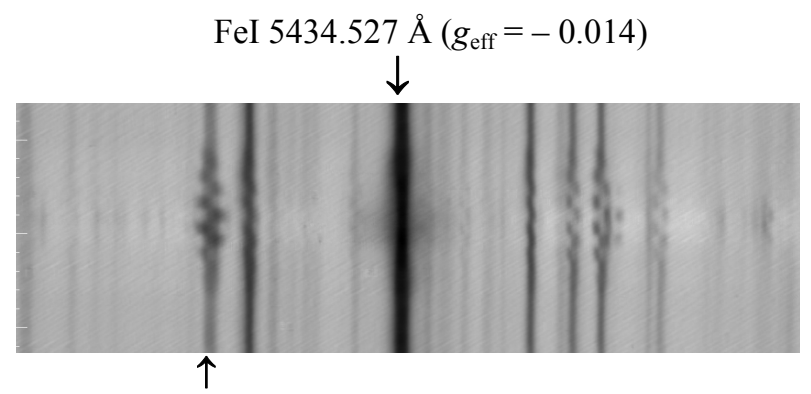

MnI $5432.548\left(g_{\text {eff }}=2.143\right)$

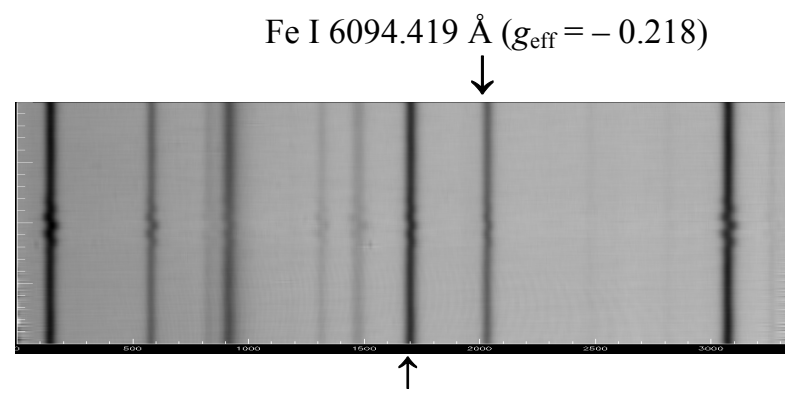

FeI $6093.65\left(g_{\text {eff }}=0.33\right)$

Figure 1: The examples of observed spectra around $5434.5 \AA$ (July 08, 2015, UT $=7^{\mathrm{h}} 30^{\mathrm{m}}$ ) and $6094.4 \AA$ (July 18, 2015, UT $=7^{\mathrm{h}} 20^{\mathrm{m}}$ ).

\section{Results}

Our main conclusions are the following:

a) as rule, spectral lines with largest Lande factors give the strongest magntic fields in sunspot umbra (Fig. 2) that can be interpreted as a result of blending the Zeeman $\pi$ - and $\sigma$-components in case of non-longitudinal magnetic field.

b) in some places of sunspots, $B_{\text {obs }}$ differs also for lines with close Lande factors, e.g. by Fe I $5432.950 \AA$ and Ni I $5435.871 \AA$ ( $g_{\text {eff }}=0.67$ and 0.5 , respectively).

c) Fe I $6094.419 \AA$ and Fe I $4995.411 \AA$ lines with $g_{\text {eff }}<0$ have in sunspots signs of the Zeeman splitting which corresponds to $g_{\text {eff }}>0$ (see, for example, Fig. 1).

Effect b) in our case can occur as a result (at least, partly) of different temperature sensitivity of lines. For example, lines Fe I $5432.950 \AA$ and Ni I 5435.871 $\AA$ have different excitation potentials of lower atomic levels, namely 4.43 and $1.98 \mathrm{eV}$, respectively.

Effect c) was discussed earlier by Lozitsky (2009). For Fe I $6094.419 \AA$ line, a wrong Lange factors is excluded because the empirically determined Lande factor $\left(g_{\text {eff }}=-\right.$ 0.218 ) is known here. So, we should search any solar effects. Two solar effects were discussed: (i) real changes of magnetic polarity at close levels $(\approx 10 \mathrm{~km})$ of solar atmosphere and (ii) Pashen-Back effect. Last effect can change of the Lande factors for Fe I lines, but in case of very strong magnetic fields $\left(\sim 10^{5} \mathrm{G}\right)$. At present, both interpretations seem as 'exotic' because they suppose extreme magnetic field changes in subtelescopic scales. In addition, such picture should be both in sunspots and outside sunspots where extremely strong magnetic fields are unlikely. True, there is one argument in favour of (ii) case. According to Solanki and Stenflo (1984), all 12 spectral lines with 'wrong' Lande factors are lines of high multiplets. Namely for such FeI lines the Pashen-Back effect is more probable than for lines of low multiplets.

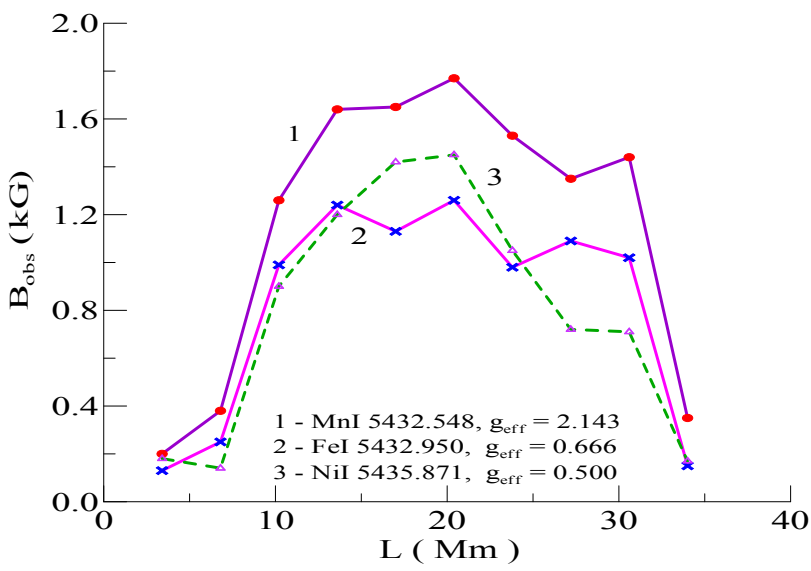

Figure 2: Comparison of observed magnetic fields $B_{\text {obs }}$ in a sunspot (AR2381, July 08, 2015) by three spectral lines with different Lande factors; $L$ - distance on the Sun surface.

As to FeI $5434.527 \AA$ line, we found very weak Zeeman-like effects in this line which were observed against a background of strong Evershed effect. Our preliminary conclusion is that this line shows spectral manifestation of the Zeeman effect in sunspot umbra which corresponds to $g_{\text {eff }}<0$.

Acknowledgements. The authors are thankful to N.I. Lozitska for helpful discussions and notes.

\section{References}

Gopasyuk S.I. et al.: 1973, Solar Phys. 31, 307.

Lozitsky V.G.: 1980, Phys. Solariterr., Potsdam, 14, 88.

Lozitsky V.G.: 2009, Bull. Crim. Astrophys. Obs. 104, No.6, 132.

Lozitsky V.G.: 2011, Int. J. Astron. Astrophys. 1 (3), 147.

Lozitsky V.G.: 2015, Adv. Space Res. 55, 958.

Semel M.: 1981, Astron. Astrophys. 97, 75.

Solanki S.K., Stenflo J.O.: 1984, Astron.Astrophys. 140, 185.

Stenflo J.O.: 1973, Solar Phys., 32, 41. 